\title{
A personal financial risk assessment intervention decreases investment fraud susceptibility in older adults
}

\author{
Matthew Martin ${ }^{a}$, Marielle C. Montenegro, Sekoul Krastev, David Brooke Struck, Jayden Rae, Dan Pilat \\ The Decision Lab
}

(Dated: February 18, 2020)

\begin{abstract}
Older adults are more often the targets and victims of financial fraud, with Americans aged 65 and older being $34 \%$ more likely to lose money due to fraud than those in their forties (Applied Research \& Consulting LLC 2013). This greater susceptibility to fraudulent offers may be due to a shift from more deliberative thinking to automatic thinking that occurs during normal ageing. This experiment tests an intervention that engages deliberative thinking about risk preferences (a Personal Risk Assessment) as a means of decreasing susceptibility to an email with tell-tale signs of a fraudulent investment. The intervention was delivered to younger (aged $<60$ ) and older (aged $60+)$ groups. Compared to age-matched controls, the intervention reduced susceptibility in the older group but not the younger one. This difference between the groups may be due to different underlying risk preferences, with the younger group having more risk tolerance. Prompting older adults to deliberately reflect on their risk-aversive preferences thus appears to counteract the effects of age on susceptibility to fraud. This experiment provides a new means of reducing susceptibility to fraud among older individuals. Future research should examine ways of decreasing susceptibility in younger populations and investigate underlying mechanisms of fraud susceptibility in both age groups.
\end{abstract}

Keywords: decision making; finance; behavioral economics; behavioral finance; stress; construal level; psychological distance

\section{INTRODUCTION}

Numerous studies have shown that older individuals are more likely to be targeted and lose money from financial fraud compared to their younger counterparts (Applied Research \& Consulting LLC 2013; Dong 2015; James et al. 2014; Shao et al. 2019; Wood and Lichtenberg 2017). Being a victim of financial fraud can have devastating consequences for those involved, including not just financial losses but also negative physical and psychological health effects including depression, anxiety, shame, and sleep disturbances (Shao et al. 2019). The annual financial loss by older adults in the United States due to financial fraud has been estimated at over $\$ 2.9$ billion dollars, and may be increasing with time (Roberto and Teaster 2011).

The internet provides a cheap and effective means of targeting and soliciting a large group of vulnerable people in fraudulent email scams. Unfortunately, many people are unable to detect tell-tale signs of fraudulent email tactics, particularly older individuals (Applied Research \& Consulting LLC 2013). Despite the increasing importance of protecting older individuals from financial fraud, few effective interventions strategies have been identified to prevent victimization (Ploeg et al. 2009). This study sought to test a simple intervention that may nudge older individuals towards detecting tell-tale signs of a fraudulent email scam.

\footnotetext{
${ }^{a}$ Correspondence should be addressed to Research Director Brook Struck at brooke@thedecisionlab.com
}

Older individuals may be more susceptible to fraud due to the cognitive effects associated with the normal process of ageing. Studies have shown that older adults tend to rely more on System 1 thinking (i.e., fast, automatic and unconscious) and less on System 2 (which leverages fluid intelligence to reason and think through problems deliberately; Peters et al. (2000)). Crystallized intelligence (knowledge and experience) increases with age, which compensates for decreases in fluid intelligence. However, crystallized intelligence starts to decline around age 70, which leads to an overall reduction in decision-making quality in the later years (Tymula et al. 2013). The shift towards using System 1 thinking may be a compensatory strategy that allows older individuals to overcome the declines in fluid intelligence.

This shift in thinking styles may be related to a number of other observed effects of ageing on financial decisionmaking. First, older adults are more affected by choice overload. In turn, they find it more difficult to navigate a proliferation of choices leading to suboptimal decisionmaking when they are overwhelmed. Specifically, adults in older age find it more challenging to ignore and sift through the irrelevant information when large amounts of information are available (Besedeš et al. 2012). Seccond, older adults have been shown to have reduced numeracy and are less apt at assessing risk such as when reasoning using numbers (e.g. about a $1 \%$ vs $0.1 \%$ risk; Best and Charness (2015)). Studies have also shown that financial literacy scores decline by about 2 percent per year after age 60 (Finke et al. 2011; Gamble et al. 2015).

Specific cognitive biases are particularly affected by ageing. Many studies report that risk aversion tends to increase with age (Grable et al. 2009; Weller et al. 2011). 
Framing effects are also stronger in older populations for both gain and loss conditions (Best and Charness 2015). Finally, older adults are more susceptible to the optimism bias, where they are more influenced by, and remember positive frames than negative ones (Agarwal et al. 2009).

The cognitive decline in deliberative decision-making associated with ageing and the shift towards using System 1 thinking may explain the increased susceptibility to financial fraud. Fraudsters often appeal to many innate biases and heuristics generated by System 1 such as social proof, reciprocity, and preference for certainty ('surethings'; Applied Research \& Consulting LLC (2013)). This study examines the effect of age on susceptibility to an email containing a number of tell-tale signs of a fraudulent investment. It also tests the effectiveness of an intervention aimed at engaging deliberative thinking about personal risk tolerance as a means of reducing susceptibility to such tactics. It was hypothesized that the intervention would be particularly effective in an older population, which tends to rely more on automatic, affective thinking rather than deliberative thinking.

\section{METHOD}

American participants $(N=102$; 56 male $)$ representing 35 U.S. States were recruited online using Amazon Mechanical Turk (MTurk). The sample was split into younger (age $\left.<60, M_{\text {age }}=35.42, n=72\right)$ and older groups (age $60+, M_{\text {age }}=64.84, n=31$ ) in order to observe age-related effects of the intervention on susceptibility to financial fraud.

All participants first completed a series of demographic questions asking about which U.S. State they lived in, their gender, total household income per year (in $\$ 10,000$ brackets from $<\$ 10,000$ to $>\$ 70,000)$ and their age. Participants were randomly assigned to either the treatment or control condition. Those in the treatment condition read a series of questions which comprise the Personal Risk Assessment, whereas those in the control condition skipped this step. The Personal Risk Assessment is a series of 13 Likert-scale questions about risk appetite and financial knowledge adapted from Davey (2005; see Appendix A). It was implemented as a means of engaging the participant in high-level deliberative thinking about financial risks, which may help older participants overcome their tendency to rely on automatic, affective thinking. The questions also serve as a measure of risk appetite, which allowed us to examine potential differences between the age groups as drivers of the intervention effect.

All participants then read an email (Figure 1) about an investment opportunity, which incorporated many of the common red flags of fraudulent pitches such as social proof, unrealistically high returns and guaranteed loss protection. The text was adapted from combining elements of two investment scam pitches rated as most appealing by over 2000 participants in Applied Research \& Consulting LLC (2013) report.

After reading the email, participants rated it on five 10-point Likert scales assessing the offer's appeal, trustworthiness, their willingness to invest in it, willingness to recommend it to a friend, and perceived riskiness (see Appendix B for Susceptibility questions).

For the Personal Risk Assessment scales, data were scored as numeric values with higher scores indicating more risk appetite. Because question 13 of the assessment presented risk preference as both a score between 0 and 100 and as discrete score ranges labeled from 1 to 7 , answers given as group values from 1 to 7 were converted to scores corresponding to the midpoint of the group range between 0 and 100. For the Susceptibility questions, a Susceptibility score was calculated by summing the five questions and dividing by the maximum score of 50. Income was coded as the midpoint of the bracket, with $>\$ 70,000$ coded as $\$ 75,000$. Following Cohen (1988), $\eta^{2}$ effect sizes are interpreted as: small $=.01$, medium $=.06$, large $=.14$. Cohen's $d$ effect sizes are interpreted as: small $=.2$, medium .5 , large $=.8$, and are interpreted regardless of whether the effect was statistically significant.

\section{RESULTS}

Because income has been shown to be a factor in susceptibility to financial fraud (James et al. 2014; Roberto and Teaster 2011), differences in income between older and younger groups were examined to test assumptions about further analyses. Additionally, previous studies have shown that females have a higher tendency to be victims of fraud (James et al. 2014; Roberto and Teaster 2011), so sex differences in Susceptibility score were also examined. An independent-samples $t$-test revealed no statistically significant difference in income between the younger and older age groups, $t(100)=-1.27, p=.209$, $M_{\mathrm{dif}}=-\$ 5802$ Cohen's $d=-0.27$, with younger participants having a small degree more income. There was also no statistically significant effect of sex on the aggregate Susceptibility score, $t(100)=1.16, p=.249, M_{\mathrm{dif}}=4.66$ Cohen's $d=0.23$, with females being a small degree more Susceptible than males. Due to the lack of significant group differences due to income and sex, these variables were not included in subsequent analyses.

In order to examine the effects of the intervention on different age groups, a $2 \times 2$ (Condition $\times$ Age group) between-subjects ANOVA was performed on the aggregate Susceptibility score. The analyses revealed a significant main effect of Condition, $F(1,98)=5.45, p=.022$, $\eta_{\text {partial }}^{2}=.053$, no significant main effect of Age group, $F(1,98)=2.29, p=.140, \eta_{\text {partial }}^{2}=.022$, power $_{\text {obs }}=$ .314 , and a marginally significant Condition $\times$ Age group 


\title{
Investment Opportunity
}

\author{
John Rollings<john@pivotal.com> \\ to me $\mathrm{V}$ \\ 4:23 PM (2 minutes ago) 色 $九$ : \\ Hello Tim, \\ We are a highly regarded and profitable Investment Management Company specializing in \\ the foreign exchange market, futures, options, commodities, stocks, bonds, real estate, \\ business startup, and many other investments.
}

\begin{abstract}
We would like to inform you of our high-yield investment program. The program pays from $2 \%$ to $3.4 \%$ daily depending on the investment plan you choose. The minimum term of investment is 180 days, after which you can either recover the sum of your initial investment or continue further participation in the project. You can also invest on a compound basis and get significant returns. We guarantee the safety of the invested amount in our company and we also pay a $5 \%$ referral commission. Throughout your engagement with our program we promise to deliver excellent customer service and answer any questions you may have regarding our business and your investments.
\end{abstract}

Please contact me at $789-788-456$ to discuss this opportunity further.

Sincerely,

John

Pivotal Investments Limited

Figure 1: The email containing many signs of a fraudulent investment opportunity.

interaction, $F(1,98)=3.03, p=.085, \eta_{\text {partial }}^{2}=.030$, power $_{\text {obs }}=.406$. It should be noted that a lack of power may obscure positive results, as observed power was always $<.80$.

An examination of the results (see Figure 2) using post-hoc tests ${ }^{1}$ showed that within the control group, the two age groups did not differ statistically significantly on their Susceptibility score, with older participants being slightly less susceptible than the younger participants, $t(44)=-.19, p=.85, M_{\mathrm{dif}}=-1.07$, Cohen's $d=-0.06)$. When examining just the younger age group, the intervention had a non-statistically significant impact, with a slight decrease due to the intervention, $t(69)=.49, p=.625, M_{\mathrm{dif}}=2.53$, Cohen's $d=0.12$. However, the intervention had a much stronger effect on the older participants, leading to a large statistically significant decrease in susceptibility in the older group, $t(29)=3.34, p=.002, M_{\mathrm{dif}}=13.75$, Cohen's $d=1.20$.

Following previous literature, this effect may be partly due to higher risk aversion in older adults. In order to examine this possibility, differences between the two age groups on the Personal Risk Assessment questions

\footnotetext{
${ }^{1}$ Note that $p$ values are not corrected for multiple comparisons.
}

in the treatment condition were examined using a series of univariate ANOVAs. An examination of the effect sizes reveals that older participants $(n=16)$ tended to score higher on questions related to risk aversion and were less confident in their ability to make financial decisions than younger participants $(n=40)$. For example, when asked to rank themselves as a percentile (0 to 100) of risk preference compared to the general population, older adults scored themselves lower than younger adults, $F(1,55)=3.70, p=.06, M_{\mathrm{dif}}=9.277$, $\eta_{\text {partial }}^{2}=0.063$. When asked what degree of risk are they were currently prepared to take with their financial decisions (from 1 to 5 ), older participants gave lower scores, $F(1,55)=3.88, p=.054, M_{\mathrm{dif}}=0.38, \eta_{\mathrm{partial}}^{2}=0.066$. When asked how much confidence they had in their ability to make good financial decisions (1 to 5$)$, older participants rated themselves as less confident, $F(1,55)=4.14$, $p=.047, M_{\text {dif }}=0.52, \eta_{\text {partial }}^{2}=0.070$. All other univariate ANOVAs were not statistically significant $(p>.10)$ and had small effect sizes $\left(\eta_{\text {partial }}^{2}<.036\right)$. Given the small sample size, these results should be interpreted as exploratory, however they are consistent with previous findings that older adults are more risk averse and have more difficulty making complex financial decisions (Agarwal et al. 2009; Finke et al. 2011). 


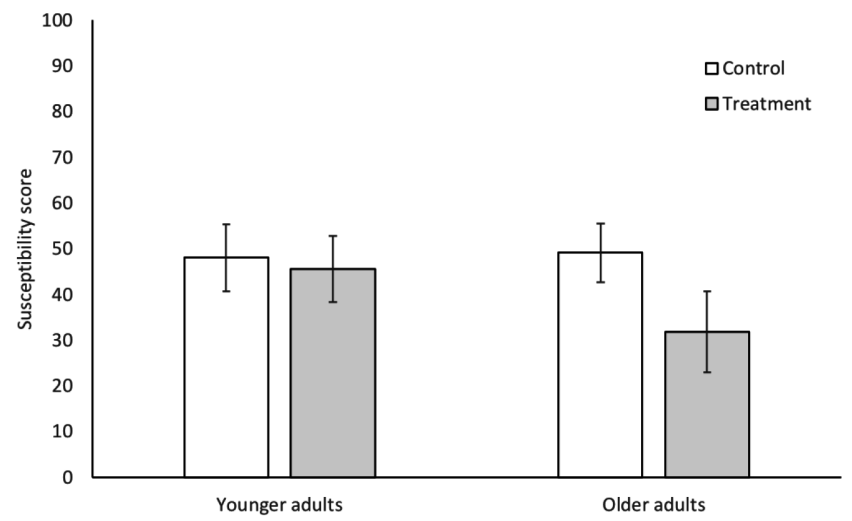

Figure 2: Effect of age group and treatment condition on susceptibility to fraudulent email.

Note. Susceptibility score is the total of the five Susceptibility questions divided by the maximum score of 50 . Error bars are $95 \%$ confidence intervals.

\section{DISCUSSION}

The study reveals that priming older adults to reflect on their risk preferences increases the likelihood they reject an investment proposal with many tell-tale fraudulent signs. The effect of the intervention was not observed for younger individuals, however. One of the causes of this difference may be due to less risk tolerance observed in the older group, consistent with previous literature. Thus, when prompted to reflect on their risk preferences, older participants would use more deliberate reasoning to reject offers that appear to violate their risk preferences. Younger participants may simply be more willing to accept a risky offer, even if they detect fraudulent signs.

Other previous research on the subject of cognitive decline and decision-making offers another possible reason why the intervention worked strictly in the older cohort. There is substantial evidence that cognitive decline affects decision-making Reed and Carstensen (2012). Earlier findings illustrate that older adults strategically change preferred decision modes (from more deliberative to more affective) in order to compensate for cognitive declines in everyday functioning. This relates to other research which has demonstrated that aging is marked by the increased salience of associative and automatic processes such as the use of heuristics (Mutter and Poliske 1994; Yates and Patalano 1999). Additionally, with the greater experience that comes with age, older adults may tend to approach tasks using more automatic processing (Myles-Worsley et al. 1988). This automatic processing can have negative consequences when applied to situations of financial decision-making.

We hypothesize that our intervention reversed the cognitive tendency of older respondents away from affective, automatic thinking towards deliberate thinking when they were faced with evaluating the investment opportu- nity. The effect was not found for younger participants, who may have been more predisposed towards using deliberative thinking by default and in turn, would be less willing to take a risky offer.

Additionally, there were no observed differences in Susceptibility scores between younger and older participants in the control group. Given previous literature suggesting that older individuals are more susceptible to financial fraud (Applied Research \& Consulting LLC 2013), one might have expected younger participants to be less susceptible to the suspicious email. One possible explanation is that the sample was recruited through MTurk, so all participants were likely fairly experienced users of technology and the internet, and so may have encountered previous fraudulent financial offers in the past. There is some evidence to suggest that accumulated experience over the course of the lifetime (wise reasoning) can counteract some of the effects of cognitive decline, which may reduce susceptibility to fraud in some cases (Grossmann et al. 2013; Shao et al. 2019).

This study had a number of limitations. First, the older participant group was smaller than the younger cohort, and the maximum participant age was 73 . The study also did not test for cognitive impairment or experience with technology, so it is unclear whether the older participants in the study were reflective of the population most susceptible to fraudulent financial proposals. A larger, older, more cognitively diverse sample would be a better test of both susceptibility to suspicious emails and the effectiveness of interventions. Second, the intervention did not appear to have much effect on the younger population. The mechanisms behind this are not clear, but may be due to differing levels of risk tolerance and default thinking styles between the age groups. Future studies should target an even older age cohort and measure cognitive decline and thinking styles to examine their effects on susceptibility to fraudulent financial emails.

The findings suggest that reflection prompts about risk preferences is an effective intervention that can prevent older adults from falling prey to fraudulent financial emails. Future studies should develop interventions that are targeted to a wider range of age groups and cognitive styles, including the young.

Sumit Agarwal, John C Driscoll, Xavier Gabaix, and David Laibson. The age of reason: Financial decisions over the life cycle and implications for regulation. Brookings $\mathrm{Pa}$ pers on Economic Activity, 2009(2):51-117, 2009. doi: https://doi.org/10.1353/eca.0.0067.

Applied Research \& Consulting LLC. Financial fraud and fraud susceptibility in the united states. research report from a 2012 national surveyâĂ Ťprepared for the finra investor education foundation., 2013. URL https://www.saveandinve 
st.org/sites/saveandinvest/files/Financial-Fraud-A nd-Fraud-Susceptibility-In-The-United-States.pdf.

Tibor Besedeš, Cary Deck, Sudipta Sarangi, and Mikhael Shor. Age effects and heuristics in decision making. Review of Economics and Statistics, 94(2):580-595, 2012. doi: https://doi.org/10.1162/REST_a_00174

Ryan Best and Neil Charness. Age differences in the effect of framing on risky choice: A meta-analysis. Psychology and aging, 30(3):688, 2015. doi:https://doi.org/10.1037/a0039447.

$\mathrm{J}$ Cohn. Statistical power analysis for the behavioral sciences (2nd ed.). Lawrence Earlbam Associates, Hillsdale, NJ, 1988.

Thomas Geoffrey Davey. Automated assessment of personal financial risk tolerance, 2005. URL http: //www.freepaten tsonline.com/6859788.html. US Patent 6,859,788.

Xin Qi Dong. Elder abuse: Systematic review and implications for practice. Journal of the American Geriatrics Society, 63(6):1214-1238, 2015. doi: https://doi.org/10.1111/jgs.13454.

Michael S Finke, John S Howe, and Sandra J Huston. Old age and the decline in financial literacy, 2011. URL https: //papers.ssrn.com/abstract=1948627. SSRN Scholarly Paper No. ID 1948627.

Keith Jacks Gamble, Patricia A Boyle, Lei Yu, and David A Bennett. Aging and financial decision making. Management Science, 61(11):2603-2610, 2015. doi: https://doi.org/10.1287/mnsc.2014.2010

John E Grable, Samantha McGill, and Sonya Britt. Risk tolerance estimation bias: The age effect. Journal of Business \&6 Economics Research (JBER), 7(7), 2009. doi: https://doi.org/10.19030/jber.v7i7.2308.

Igor Grossmann, Jinkyung Na, Michael EW Varnum, Shinobu Kitayama, and Richard E Nisbett. A route to wellbeing: Intelligence versus wise reasoning. Journal of Experimental Psychology: General, 142(3):944, 2013. doi: https://doi.org/10.1037/a0029560

Bryan D James, Patricia A Boyle, and David A Bennett. Correlates of susceptibility to scams in older adults without dementia. Journal of elder abuse \& neglect, 26(2):107-122, 2014. doi:https://doi.org/10.1080/08946566.2013.821809.

Sharon A Mutter and Rebecca M Poliske. Aging and illusory correlation in judgments of co-occurrence. Psychology and aging, 9(1):53-63, 1994. doi:https://doi.org/10.1037/08827974.9.1.53.
Marina Myles-Worsley, William A Johnston, and Margaret A Simons. The influence of expertise on x-ray image processing. Journal of Experimental Psychology: Learning, Memory, and Cognition, 14(3):553-557, 1988 . doi: https://doi.org/10.1037/0278-7393.14.3.553.

Ellen Peters, Melissa L Finucane, Donald G MacGregor, and Paul Slovic. The bearable lightness of aging: Judgment and decision processes in older adults. The aging mind: Opportunities in cognitive research, pages 144-165, 2000.

Jenny Ploeg, Jana Fear, Brian Hutchison, Harriet MacMillan, and Gale Bolan. A systematic review of interventions for elder abuse. Journal of Elder Abuse \& Neglect, 21(3):187-210, 2009. doi:https://doi.org/10.1080/08946560902997181.

Andrew E Reed and Laura L Carstensen. The theory behind the age-related positivity effect. Frontiers in psychology, 3: 339, 2012. doi:https://doi.org/10.3389/fpsyg.2012.00339

Karen Roberto and Pamela Teaster. The metlife study of elder financial abuse: Crimes of occasion, desperation, and predation against america's elders. 2011. URL https://nc vc.dspacedirect.org/handle/20.500.11990/641.

Jingjin Shao, Qianhan Zhang, Yining Ren, Xiying Li, and Tian Lin. Why are older adults victims of fraud? current knowledge and prospects regarding older adultsâĂŹ vulnerability to fraud. Journal of elder abuse $\&$ neglect, 31(3):225-243, 2019. URL https://doi.org/10.1080/ 08946566.2019 .1625842 .

Agnieszka Tymula, Lior A Rosenberg Belmaker, Lital Ruderman, Paul W Glimcher, and Ifat Levy. Like cognitive function, decision making across the life span shows profound age-related changes. Proceedings of the National Academy of Sciences, 110(42):17143-17148, 2013. doi:17143âĂŞ17148. https://doi.org/10.1073/pnas.1309909110.

Joshua A Weller, Irwin P Levin, and Natalie L Denburg. Trajectory of risky decision making for potential gains and losses from ages 5 to 85. Journal of Behavioral Decision Making, 24 (4):331-344, 2011. doi:https://doi.org/10.1002/bdm.690

Stacey Wood and Peter A Lichtenberg. Financial capacity and financial exploitation of older adults: Research findings, policy recommendations and clinical implications. Clinical gerontologist, 40(1):3-13, 2017. doi: https://doi.org/10.1080/07317115.2016.1203382

J Frank Yates and Andrea L Patalano. Decision making and aging. In In Processing of medical information in aging patients: Cognitive and human factors perspectives, pages 3154. Mahwah, NJ, US: Lawrence Erlbaum Associates Publishers, 1999. 
Appendix A: Personal Risk Assessment

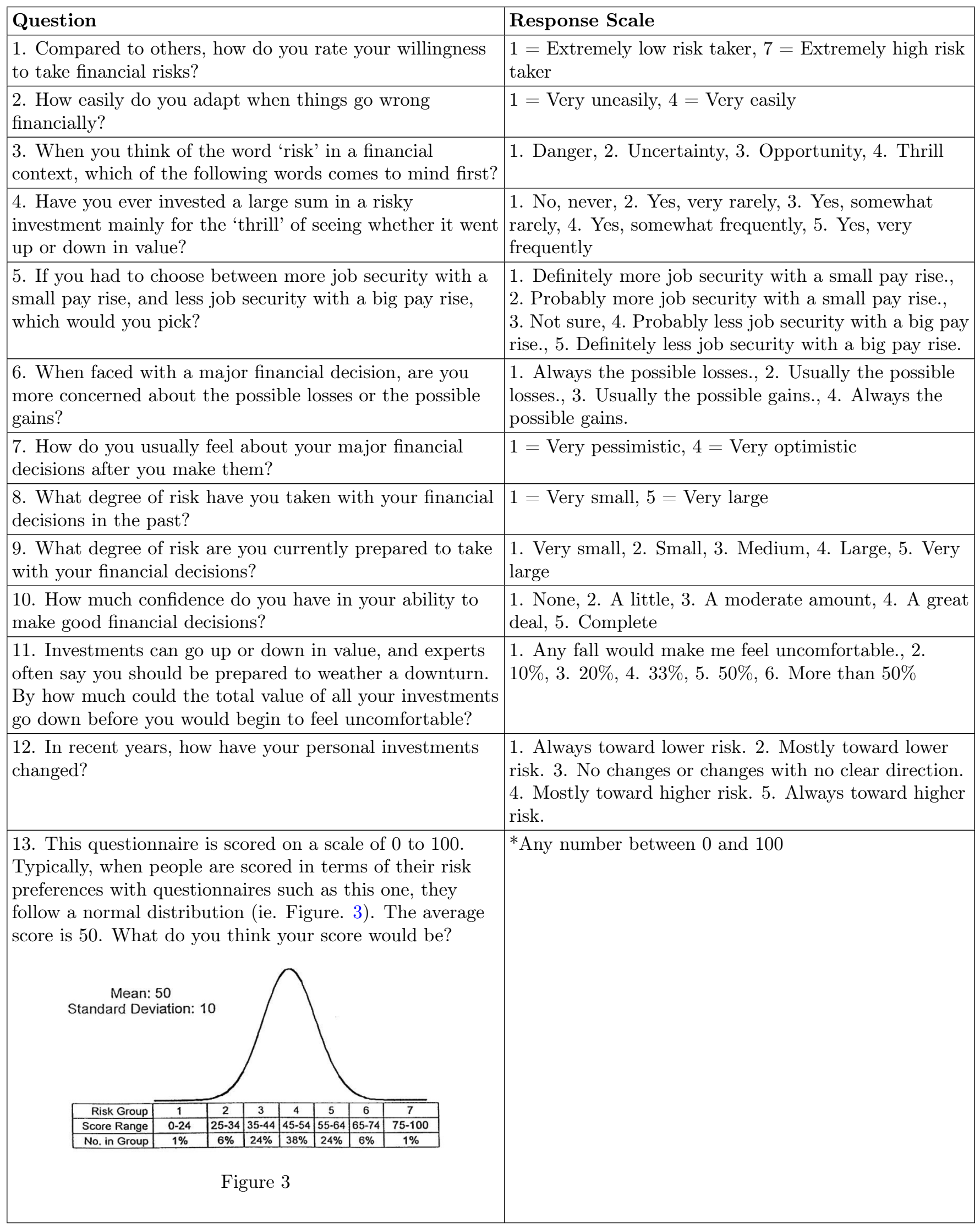




\section{Appendix B: Susceptibility Questionnaire}

\begin{tabular}{|l|l|}
\hline Question & Response Scale \\
\hline 1. I find this potential investment opportunity appealing. & $1=$ Strongly disagree, $10=$ Strongly agree \\
\hline 2. I think the investment company is trustworthy. & $1=$ Strongly disagree, $10=$ Strongly agree \\
\hline 3. I would like to invest in this investment opportunity. & $1=$ Strongly disagree, $10=$ Strongly agree \\
\hline 4. I would recommend this investment opportunity to a friend. & $1=$ Strongly disagree, $10=$ Strongly agree \\
\hline 5. I think this is a risky investment opportunity. & $1=$ Strongly disagree, $10=$ Strongly agree \\
\hline
\end{tabular}

